\title{
Resveratrol attenuates type 2 diabetes mellitus by mediating mitochondrial biogenesis and lipid metabolism via Sirtuin type 1
}

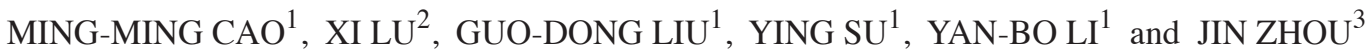 \\ Departments of ${ }^{1}$ Endocrinology, ${ }^{2}$ Gastroenterology and ${ }^{3}$ Hematology, The First Affiliated \\ Hospital of Harbin Medical University, Harbin, Heilongjiang 150001, P.R. China
}

Received January 4, 2017; Accepted August 24, 2017

DOI: $10.3892 /$ etm.2017.5400

\begin{abstract}
The rising incidence of type 2 diabetes mellitus (T2DM) is a major public health problem and novel therapeutic strategies are required to prevent and treat T2DM. It has been demonstrated that resveratrol (RSV) may prevent T2DM by targeting Sirtuin type 1 (SIRT1), indicating that SIRT1 may be a novel therapeutic target for T2DM prevention. In the present study, a T2DM rat model was established by administering a high fat diet and streptozotocin (STZ) injections. Measurements of blood glucose and insulin confirmed successful establishment of the T2DM model. RSV was used to treat rats with STZ-induced T2DM and the results indicated that RSV reversed the STZ-induced downregulation of peroxisome proliferator-activated receptor- $\gamma$ coactivator- $1 \alpha$, SIRT1 and forkhead box protein O 3a. Furthermore, RSV modulated the activity of superoxide dismutase and malondialdehyde, which are associated with oxidative stress. In vitro, cells from the insulinoma cell line clone $1 \mathrm{E}$ were pretreated with palmitic acid (PA) to simulate a high fat environment. The results of reverse transcription-quantitative polymerase chain reaction indicated that PA suppressed the expression of SIRT1 in a dose- and time-dependent manner. Furthermore, PA modulated the expression of mitochondrial biogenesis-associated, lipid metabolism-associated and $\beta$-cell-associated genes,
\end{abstract}

Correspondence to: Dr Yan-Bo Li, Department of Endocrinology, The First Affiliated Hospital of Harbin Medical University, 199 Dazhi Street, Nangang, Harbin, Heilongjiang 150001, P.R. China E-mail: 1i_yanboo@sina.cn

Abbreviations: T2DM, type 2 diabetes mellitus; NRF, nuclear respiratory factor; mtTFA, mitochondrial transcription factor A; CPT-1, lipid metabolism-related carnitine palmitoyltransferase 1; ACC, acetyl-CoA carboxylase; LCAD, long-chain acyl-CoA dehydrogenase; PDX-1, pancreatic duodenal homeobox-1 gene; MAFA, mid-arm fat area; INS, insulin; RSV, resveratrol; STZ, streptozotocin; INS-1E, insulinoma cell line clone 1E; SIRT1, Sirtuin type 1; PGC-1 $\alpha$, peroxisome proliferator-activated receptor- $\gamma$ coactivator- $1 \alpha$; FOXO, forkhead box O; PA, palmitic acid

Key words: sirtuin type 1, resveratrol, type 2 diabetes mellitus, lipid metabolism, mitochondrial biogenesis, insulin resistance whereas RSV treatment ameliorated the PA-induced changes in the expression of these genes via SIRT1. The results of the present study suggest that RSV participates in the prevention of T2DM by regulating the expression of mitochondrial genes associated with biogenesis, lipid metabolism and $\beta$-cells via SIRT1. The results of the current study provide an insight into the mechanisms by which SIRT1 inhibits T2DM and may be used as a basis for future studies.

\section{Introduction}

Due to rapid economic development and continuous improvements in living standards, the prevalence of diabetes is increasing worldwide (1). Type 2 diabetes mellitus (T2DM) is a complex metabolic disorder caused by insulin (INS) resistance and insufficient compensatory INS secretion (2). T2DM greatly increases the risk of chronic kidney disease, cardiovascular disease, myocardial infarction and stroke (3); therefore, more effective treatments and novel prevention strategies for T2DM are required (4). The mechanisms of T2DM action, including lipid metabolism disorders, endothelial dysfunction, chronic inflammation and redox homeostasis imbalance, have previously been described (5). $\beta$-cells dysfunction contributes to inadequate INS secretion, which is the ultimate cause of T2DM (6). It has been demonstrated that high levels of free fatty acids or hypertriglyceridemia and lipid ectopic deposition in non adipose tissue are key factors contributing to the development of T2DM (7). Total $\beta$-cells function is determined by the number of $\beta$-cellss and their functionality. Establishing an effective therapy to decrease $\beta$-cells lipid toxicity may be beneficial for the treatment of T2DM.

The progression of T2DM is associated with changes in several factors, including mitochondrial biogenesis, lipid metabolism and $\beta$-cells development (5). A number of proteins, including mitochondrial biogenesis-related nuclear respiratory factor (NRF), mitochondrial transcription factor A (mtTFA), lipid metabolism-associated carnitine palmitoyltransferase 1 (CPT-1), acetyl-CoA carboxylase (ACC), long-chain acyl-CoA dehydrogenase (LCAD), $\beta$-cells-associated pancreatic duodenal homeobox-1 gene (PDX-1), mid-arm fat area (MAFA) (8) and INS, are associated with T2DM (9). Resveratrol (RSV), a natural polyphenol found in grapes and red wine (10), may potentially attenuate hepatic steatosis and reduce the risk of patients developing T2DM (11). It has previously been demonstrated 
that RSV activates Sirtuin type 1 (SIRT1) to inhibit the development of T2DM (12). SIRT1 is a nuclear NAD ${ }^{+}$-dependent class III histone deacetylase that may regulate cell aging, life span and energy metabolism (13) by engaging in the reciprocal co-regulation of different binding partners (14). SIRT1 is able to adjust the deacetylase activity of various transcription factors that control metabolic and endocrine signals and is widely involved in regulating the mammalian cell life span, INS secretion and glucose/lipid metabolism (15). Genetic variation in the SIRT1 gene is associated with INS resistance and may explain the increased risk of T2DM in the Chinese Han population (16). SIRT1 may have a therapeutic effect on metabolic deterioration in T2DM (17) and in the liver, SIRT1-knockout increases plasma glucose levels, reduces INS sensitivity and upregulates free fatty acid and cholesterol levels (18). To explore the association between SIRT1 and mitochondrial biogenesis, it is essential to assess lipid metabolism and $\beta$-cells development and identify the underlying mechanisms by which RSV alleviates T2DM.

In the present study, a T2DM rat model was constructed following the administration of a high fat diet and streptozotocin (STZ) injections into the abdominal cavity of rats. It was determined that the T2DM model was successfully constructed following the assessment of glucose and INS levels. The results indicated that RSV treatment effectively modulated glucose and INS levels as well as the expression of SIRT1, peroxisome proliferator-activated receptor- $\gamma$ coactivator- $1 \alpha$ (PGC-1 $\alpha$ ), and forkhead box protein O3 (FOXO3a). In addition, the insulinoma cell line clone 1E (INS-1E) was treated with palmitic acid (PA). PA suppressed the expression of SIRT1 in a dose- and time-dependent manner. In PA-induced INS-1E cells, it was demonstrated that RSV modulated the expression of PGC-1 $\alpha$ and FOXO3a via SIRT1 and regulated the expression of mitochondrial biogenesis-associated, lipid metabolism-associated and $\beta$-cells-associated proteins. These results provide novel evidence that RSV mitigates T2DM via SIRT1 and identified the mechanisms by which SIRT1 inhibits T2DM.

\section{Materials and methods}

Animals. A total of 30 male Sprague-Dawley (SD) rats (6-8 weeks old) weighing 200 $\pm 20 \mathrm{~g}$ were purchased from Hunan SJA Laboratory Animal Co., Ltd. (Changsha, China). Rats were housed at $24 \pm 1^{\circ} \mathrm{C}$ with $40-50 \%$ humidity in a clean environment with a $12 \mathrm{~h}$ light/dark cycle. All animals had free access to food and purified water. All procedures were approved by the Animal Ethics Committee of the First Affiliated Hospital of Harbin Medical University (Harbin, China). Rats were fed with a high glucose, high fat diet (60\% common chow, $10 \%$ lard, $10 \%$ egg yolk powder and $20 \%$ sucrose) for 8 weeks. Rats then received a $40 \mathrm{mg} / \mathrm{kg}$ intraperitoneal injection of STZ (Sigma-Aldrich; Merck KGaA, Darmstadt, Germany) once. At $72 \mathrm{~h}$ following STZ injection, rats with fasting blood glucose $\geq 16.67 \mathrm{mM}$ (based on tail vein samples) were selected as T2DM animals for use in the following experiments (19). The normal control group (NC, $\mathrm{n}=10$ ) was administered the same volume of sodium citrate buffer instead of STZ. T2DM rats were randomly assigned to either a diabetic group (DM, $n=10$ ) or a diabetic RSV group (DR, $n=10)$. Rats in the DR group were administered once with $30 \mathrm{mg} / \mathrm{kg}$ RSV intragastrically (Sigma-Aldrich; Merck $\mathrm{KGaA})$. Rats in the $\mathrm{NC}$ and DM groups were administered intragastrically with the same amount of $0.5 \%$ carboxymethyl cellulose. At weeks 0,4 and 8 following induction, fasting and $2 \mathrm{~h}$ postprandial blood glucose were measured using the glucose oxidase method on the Beckman Glucose Analyzer II (Beckman Coulter, Inc., Brea, CA, USA) and plasma INS levels were determined using an RIA Assay system (Linco Research, Inc., St. Charles, MO, USA) (20) according to the manufacturer's protocol. Following 8 weeks of different treatments, total RNA was isolated from rat pancreas tissues using TRIzol reagent (Invitrogen; Thermo Fisher Scientific, Inc., Waltham, MA, USA), and proteins were extracted using radioimmunoprecipitation assay buffer (RIPA; Beyotime Institute of Biotechnology, Haimen, China) for western blot analysis.

Cell culture and grouping. The rat insulinoma cell line clone 1E (INS-1E; provided by Professor Pierre Maechler; CMU; University of Geneva, Geneva, Switzerland) was cultured at $37^{\circ} \mathrm{C}$ in a humidified atmosphere containing $5 \% \mathrm{CO}_{2}$ in RPMI 1640 medium (HyClone; GE Healthcare Life Sciences, Logan, UT, USA) supplemented with $10 \%$ fetal bovine serum (FBS; Sigma-Aldrich; Merck KGaA), 11 mM glucose, $10 \mathrm{mM}$ HEPES, $1 \mathrm{mM}$ sodium pyruvate, $50 \mu \mathrm{M}$ 2-mercaptoethanol (Sigma-Aldrich; Merck KGaA), $50 \mu \mathrm{g} / \mathrm{ml}$ penicillin and $100 \mu \mathrm{g} / \mathrm{ml}$ streptomycin. When density reached $80 \%$, cells were pretreated with PA $(0,0.125,0.25,0.5$, or $1 \mathrm{mM})$ for $24 \mathrm{~h}$. INS-1E cells were treated with $0.5 \mathrm{mM}$ PA for $0,12,24,48$ or $72 \mathrm{~h}$.

Cell transfection. INS-1E cells were seeded into 6-well plates at a density of $5 \times 10^{5}$ cells/well and divided into 5 groups: A normal control (NC) group, a high fat (HF) group, a HF+RSV group, a HF+RSV+SIRT small interfering (si)RNA group (HF+RSV+SIRT siRNA) and a HF+RSV+negative control siRNA group (HF+RSV+NC siRNA). To transfect cells with SITR siRNA or NC siRNA, cells in the HF+RSV+SIRT siRNA and $\mathrm{HF}+\mathrm{RSV}+\mathrm{NC}$ siRNA groups were incubated in $1 \mathrm{ml}$ RPMI 1640 with SIRT1 siRNA (5'-CACCCCAGCAAC TCAGCATTCATCGAAATGAATGCTGAGTTGCTGG-3') or NC siRNA (5'-TTCTCCGAACGTGTCACGT-3'), respectively, and Lipofectamine 2000 (Thermo Fisher Scientific, Inc.) without FBS. The final concentrations of siRNA and Lipofectamine 2000 used were 2 and $4 \mu \mathrm{g} / \mathrm{ml}$, respectively. Following $48 \mathrm{~h}$ incubation at $37^{\circ} \mathrm{C}$, cells in the $\mathrm{HF}+\mathrm{RSV}$, $\mathrm{HF}+\mathrm{RSV}+\mathrm{SIRT}$ siRNA and $\mathrm{HF}+\mathrm{RSV}+\mathrm{NC}$ siRNA groups were treated with $0.5 \mathrm{mM} \mathrm{PA}+10 \mu \mathrm{M} \mathrm{RSV}$. The HF group received the same amount of PA and NC cells were treated with the same amount of vehicle. Following $24 \mathrm{~h}$ incubation at $37^{\circ} \mathrm{C}$, total RNA or proteins were extracted for further experiments.

Reverse transcription-quantitative polymerase chain reaction $(R T-q P C R)$. Total RNA was isolated from INS-1E cells using TRIzol reagent (Invitrogen; Thermo Fisher Scientific, Inc.) following the manufacturer's protocol. The quality of RNA was determined by agarose electrophoresis and the concentration was measured using a NanoDrop ND-2000 spectrophotometer (Thermo Fisher Scientific, Inc., Pittsburgh, PA, USA) at $260 \mathrm{~nm}$ and $280 \mathrm{~nm}$. RT was performed using $1 \mu \mathrm{g}$ total RNA using a reverse transcriptase cDNA synthesis 
Table I. Primers used for reverse transcription-quantitative polymerase chain reaction.

\begin{tabular}{|c|c|c|}
\hline Genes & Forward primers $\left(5^{\prime}-3^{\prime}\right)$ & Reverse primers $\left(5^{\prime}-3^{\prime}\right)$ \\
\hline SIRT1 & GCTGACGACTTCGACGACG & TCGGTCAAGAGGAGGTTGTCT \\
\hline$P G C 1 \alpha$ & TATGGAGTGACATAGAGTGTGCT & GTCGCTACACCACTTCAATCC \\
\hline$N R F$ & CGGAAACGGCCTCATGTGT & CGCGTCGTGTACTCATCCAA \\
\hline FOXO3a & TCTTACGCCGACCTCATCAC & ACGCTCTTGACCATCCACT \\
\hline INS & CCCTGTTGGTGCACTTCCT & TCCCAGCTCCAGTTGTTCC \\
\hline$m t T F A$ & TATTAGAATTTGTTAAATTTTGGGGAAT & ACAАACAАТССТАСАТССАAААСC \\
\hline$A C C$ & CACATCATGAAGGAGGAGG & GCTATCACACAGCCTGGGTC \\
\hline$C P T-1$ & ACGGGTGGATGTTCGAGATG & GGCAGTGACGTTTGGAAGCT \\
\hline$L C A D$ & TTCGTGTCCTGAGCG & GAGGCTAATGCCATG \\
\hline$P D X-1$ & GTTCATCTCCCTTTCCCGTGG & GGTGGTGGCTTTGGCAATG \\
\hline$M A F A$ & AAAGCGGTGCTGGAGGAT & GGTTCAGGTGGTGCTGGTA \\
\hline$\beta$-actin & ССТCTATGCCAACACAGTGC & GTACTCCTGCTTGCTGATCC \\
\hline
\end{tabular}

SIRT1, Sirtuin 1; PGC1 $\alpha$, peroxisome proliferator-activated receptor- $\gamma$ coactivator-1 $\alpha$; NRF, nuclear respiratory factor; FOXO3a, forkhead box O3a; INS, insulin; mtTFA, mitochondrial transcription factor A; ACC, acetyl-CoA carboxylase; CPT-1, lipid metabolism-related carnitine palmitoyltransferase 1; LCAD, long-chain acyl-CoA dehydrogenase; PDX-1, pancreatic duodenal homeobox-1; MAFA, mid-arm fat area.

kit (Takara Biotechnology Co., Ltd., Dalian, China) according to the manufacturer's protocol. qPCR was performed using SYBR-Green qPCR SuperMix (Invitrogen; Thermo Fisher Scientific, Inc.) on a real-time RT-PCR system (Bio-Rad Laboratories, Inc., Hercules, CA, USA). The thermocycling conditions were as follows: $94^{\circ} \mathrm{C}$ for $5 \mathrm{~min}$ followed by 35 cycles of $94^{\circ} \mathrm{C}$ for $30 \mathrm{sec}, 56^{\circ} \mathrm{C}$ for $30 \mathrm{sec}$ and $72^{\circ} \mathrm{C}$ for $30 \mathrm{sec}$, with a final elongation step at $72^{\circ} \mathrm{C}$ for $5 \mathrm{~min}$. All amplification reactions were performed in triplicate and the relative expression of target genes were normalized against $\beta$-actin using the $2^{-\Delta \Delta \mathrm{Cq}}$ method (21). The primers for RT-qPCR are presented in Table I.

Western blotting. Proteins were extracted from rat pancreatic tissues or INS-1E cells using RIPA buffer (Beyotime Institute of Biotechnology, Haimen, China) and centrifuged at $15,000 \mathrm{x} \mathrm{g}$ for $30 \mathrm{~min}$ at $4^{\circ} \mathrm{C}$. The concentration of proteins was determined using a bicinchoninic acid kit (Pierce; Thermo Fisher Scientific, Inc.), according to the manufacturer's protocol. Equal amounts of protein $(25 \mu \mathrm{g} / \mathrm{lane})$ were subjected to $12.5 \%$ SDS-PAGE. Proteins were subsequently electrotransferred to a polyvinylidene fluoride membrane and blocked with $5 \%$ non-fat milk in Tris-buffered saline with $0.05 \%$ Tween-20 (TBST) for $1 \mathrm{~h}$ at room temperature. The membrane was incubated with mouse anti-SIRT1 (sc-15404), anti-FOXO3a (sc-20680), anti-PGC-1 $\alpha$ (sc-13067) and anti- $\beta$-actin (sc-1616) antibodies (all 1:1,000 dilution; all from Santa Cruz Biotechnology, Inc., Dallas, TX, USA) overnight at $4^{\circ} \mathrm{C}$. Following three washes with TBST, the membrane was incubated with horseradish peroxidase-conjugated rabbit anti-mouse secondary antibody (1:2,000; sc-203; Santa Cruz Biotechnology, Inc.) for $1 \mathrm{~h}$ at room temperature. Membrane bands were developed using an enhanced chemiluminescence kit (Pierce; Thermo Fisher Scientific, Inc.). The relative densities of target protein bands were normalized against $\beta$-actin using Quantity One software v4.4 (Bio-Rad Laboratories, Inc.).
Detection of serum superoxide dismutase (SOD) and malondialdehyde (MDA). Total heart blood was harvested from rats following 8 weeks of different treatments and centrifuged at $3,500 \mathrm{x} g$ for $15 \mathrm{~min}$ at $4^{\circ} \mathrm{C}$ to separate the serum. Serum SOD (cat. no. SES134Hu) and MDA (cat. no. CEA597Ge) levels were detected using a commercially available sandwich ELISA kit (Cloud-Clone Corp., Katy, TX, USA) according to the manufacturer's protocol. Absorbance was measured at $450 \mathrm{~nm}$ on Bio-Rad 680 Microplate reader (Bio-Rad Laboratories, Inc.). The quantity of SOD and MDA in the serum was estimated using a constructed calibration curve.

Statistical analysis. All statistical analyses were performed using SPSS 17.0 (SPSS, Inc., Chicago, IL, USA). All results are presented as the mean \pm standard error of the mean. Significant differences in mean values were evaluated using one-way analysis of variance with Tukey's post hoc test. $\mathrm{P}<0.05$ was considered to indicate a statistically significant difference.

\section{Results}

$R S V$ treatment decreases blood glucose and INS levels in rats with STZ-induced DM. To confirm that the diabetic rat model was successfully established, blood glucose levels were measured following STZ injection. No significant differences in blood glucose levels were observed in the $\mathrm{NC}$ group at weeks 0,4 or 8 (Table II). Compared with the NC group, fasting glucose levels in the DM group were significantly higher at week $8(\mathrm{P}<0.05$; Table II). However, the administration of RSV significantly attenuated the STZ-induced increase in fasting glucose $(\mathrm{P}<0.05$; Table II). The $2 \mathrm{~h}$ postprandial blood glucose levels were subsequently assessed. At weeks 4 and 8 post-induction, postprandial blood glucose was significantly higher in the DM group compared with the NC group $(\mathrm{P}<0.01$; Table II); however, in the DR group, this increase was significantly attenuated $(\mathrm{P}<0.01$; Table II). 
Table II. Fasting and $2 \mathrm{~h}$ postprandial blood glucose levels at weeks 0,4 and 8 following induction of diabetes.

\begin{tabular}{lccc}
\hline & & \multicolumn{2}{c}{ Blood glucose $(\mathrm{mM})$} \\
\cline { 3 - 4 } Group & Time (weeks) & Fasting & 2 h postprandial \\
\hline NC & 0 & $5.86 \pm 0.51$ & $6.14 \pm 0.62$ \\
& 4 & $5.91 \pm 0.64$ & $6.24 \pm 0.64$ \\
& 8 & $5.74 \pm 0.49$ & $6.05 \pm 0.59$ \\
DM & 0 & $5.79 \pm 0.41$ & $6.11 \pm 0.61$ \\
& 4 & $6.45 \pm 0.62$ & $15.98 \pm 0.71^{\mathrm{b}}$ \\
\multirow{2}{*}{ DR } & 8 & $7.97 \pm 0.71^{\mathrm{a}}$ & $22.36 \pm 1.14^{\mathrm{b}}$ \\
& 0 & $5.84 \pm 0.47$ & $6.31 \pm 0.61$ \\
& 4 & $6.24 \pm 0.59$ & $12.36 \pm 0.89^{\mathrm{d}}$ \\
& 8 & $7.01 \pm 0.68^{\mathrm{c}}$ & $13.59 \pm 0.97^{\mathrm{d}}$ \\
\hline
\end{tabular}

${ }^{\mathrm{a}} \mathrm{P}<0.05$ and ${ }^{\mathrm{b}} \mathrm{P}<0.01$ vs. NC group; ${ }^{\mathrm{C}} \mathrm{P}<0.05$ and ${ }^{\mathrm{d}} \mathrm{P}<0.01$ vs. $\mathrm{DM}$ group. NC, normal control group; DM, streptozotocin-induced diabetic group; DR, RSV treated streptozotocin-induced diabetic group.

Table III. Fasting and $2 \mathrm{~h}$ postprandial blood insulin levels at weeks 0,4 and 8 following induction of diabetes.

\begin{tabular}{lccc}
\hline \multirow{2}{*}{ Group } & Time (weeks) & \multicolumn{2}{c}{ Blood insulin (ng/ml) } \\
\cline { 3 - 4 } NC & 0 & $1.21 \pm 0.14$ & $1.14 \pm 0.07$ \\
& 4 & $1.24 \pm 0.14$ & $1.16 \pm 0.09$ \\
& 8 & $1.29 \pm 0.49$ & $1.15 \pm 0.09$ \\
DM & 0 & $1.25 \pm 0.11$ & $1.11 \pm 0.06$ \\
& 4 & $1.45 \pm 0.12^{\mathrm{a}}$ & $1.37 \pm 0.11^{\mathrm{a}}$ \\
& 8 & $1.56 \pm 0.11^{\mathrm{a}}$ & $1.47 \pm 0.14^{\mathrm{a}}$ \\
$\mathrm{DR}$ & 0 & $1.24 \pm 0.07$ & $1.17 \pm 0.09$ \\
& 4 & $1.32 \pm 0.09$ & $1.35 \pm 0.09$ \\
& 8 & $1.35 \pm 0.08^{\mathrm{b}}$ & $1.37 \pm 0.07^{\mathrm{b}}$ \\
\hline
\end{tabular}

${ }^{\mathrm{a}} \mathrm{P}<0.05$ vs. NC group and ${ }^{\mathrm{b}} \mathrm{P}<0.05$ vs. $\mathrm{DM}$ group. $\mathrm{NC}$, normal control group; DM, streptozotocin-induced diabetic group; DR, RSV treated streptozotocin-induced diabetic group.

The fasting and $2 \mathrm{~h}$ postprandial levels of INS were also assessed. Compared with the NC group, fasting and $2 \mathrm{~h}$ postprandial blood INS levels were significantly elevated in the DM group at weeks 4 and $8(\mathrm{P}<0.05$; Table III). However, INS levels in the DR group were significantly lower at week 8 compared with the DM group $(\mathrm{P}<0.05$; Table III). Taken together, these results suggest that STZ injection successfully induced DM in rats and that RSV administration modulated glucose and INS levels in rats with STZ-induced DM.

RSV modulates the expression of PGC-1 $\alpha$, SIRT1 and FOXO3a in the pancreatic tissues of rats with STZ-induced DM. To investigate the mechanism by which RSV functions in DM, levels of PGC-1 $\alpha$, SIRT1 and FOXO3a mRNA and protein were assessed using RT-qPCR and western blotting, respectively.
Compared with the NC group, levels of PGC-1 $\alpha$, SIRT1 and FOXO3a mRNA and protein were significantly suppressed in the DM group $(\mathrm{P}<0.01$; Fig. $1 \mathrm{~A}$ and $\mathrm{B})$. However, pretreatment with RSV significantly attenuated these decreases $(\mathrm{P}<0.05$; Fig. 1A and B). These data suggest that RSV administration upregulates PGC-1 $\alpha$, SIRT1 and FOXO3a expression in mice with STZ-induced DM.

It has been reported that oxidawtive stress due to the excessive production of reactive oxygen species (ROS) and mitochondrial dysfunction are major factors in the development of INS resistance in T2DM (22). ELISA assays were used to analyze the activity of SOD and MDA to evaluate oxidative stress. SOD activity was significantly suppressed in the DM group compared with the NC group $(\mathrm{P}<0.01$; Fig. 1C). However, RSV treatment significantly increased SOD activity in the DR group compared with the DM group $(\mathrm{P}<0.05$; Fig. $1 \mathrm{C})$. By contrast, MDA activity was significantly upregulated in the $\mathrm{DM}$ group compared with the NC group ( $\mathrm{P}<0.01$; Fig. 1D). In the DR group, pretreatment with RSV significantly attenuated the DM-induced increase in MDA activity ( $\mathrm{P}<0.05$; Fig. 1D). These data suggest that RSV regulates the activity of SOD and MDA in rats with STZ-induced DM.

PA inhibits the expression of SIRT1 in a dose-and time-dependent manner. It has been demonstrated that PA serves a role in the development of obesity and T2DM (17). Varying concentrations of PA were used to treat INS-1E cells and the results indicated that PA significantly suppressed SIRT1 mRNA in a dose-dependent manner ( $\mathrm{P}<0.01$; Fig. $2 \mathrm{~A})$. The association between SIRT1 expression and PA induction time was subsequently investigated and the results indicate that PA treatment also significantly reduced SIRT1 mRNA expression in a time-dependent manner $(\mathrm{P}<0.01$; Fig. $2 \mathrm{~B})$.

$R S V$ affects the expression of PGC-1 $\alpha$ and FOXO3a via SIRT1 in PA-induced INS-IE cells. To further investigate the role of RSV in vitro, levels of PGC- $1 \alpha$, SIRT1 and FOXO3a in INS-1E cells were evaluated. PA significantly suppressed the levels of PGC-1 $\alpha$, SIRT1 and FOXO3a mRNA and protein compared with the NC group $(\mathrm{P}<0.01$; Fig. 3A-C); however, administration of RSV significantly attenuated this effect $(\mathrm{P}<0.01$; Fig. 3A-C). Furthermore, transfection with SIRT1 siRNA in conjunction with PA significantly reduced the expression of PGC-1 $\alpha$, SIRT1 and FOXO3a compared with the $\mathrm{HF}+\mathrm{RSV}+\mathrm{NC}$ siRNA group $(\mathrm{P}<0.01$; Fig. 3A-C). These data suggest that RSV mitigates the HF-induced inhibition of PGC- $1 \alpha$ and FOXO3a expression via SIRT1.

To examine the mechanism by which SIRT1 functions, the expression of a cascade of genes was investigated, including mitochondrial biogenesis-associated NRF and mtTFA, lipid metabolism-associated CPT-1, ACC and LCAD, and $\beta$-cells-associated PDX-1, MAFA and INS. PA treatment significantly suppressed mRNA levels of SIRT1, NRF, mtTFA, CPT-1, LCAD, PDX-1, MAFA and INS mRNA, whereas it significantly upregulated the expression of ACC mRNA compared with the NC group $(\mathrm{P}<0.01$; Fig. 4). Treatment with RSV significantly attenuated these PA-induced alterations in gene expression $(\mathrm{P}<0.01$; Fig. 4). However, SIRT1 knockdown significantly attenuated the PA-induced increases in SIRT1, NRF, mtTFA, CPT-1, LCAD, 
A

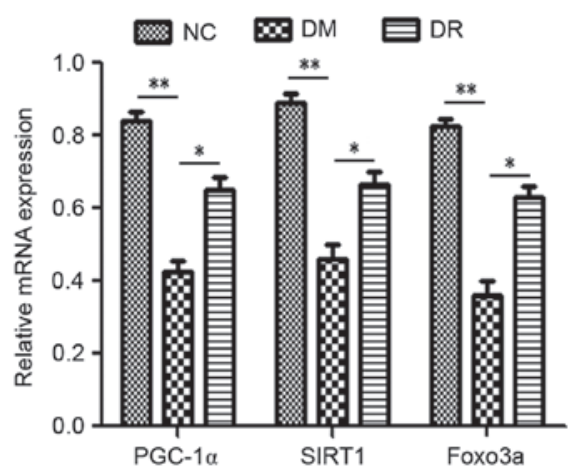

C

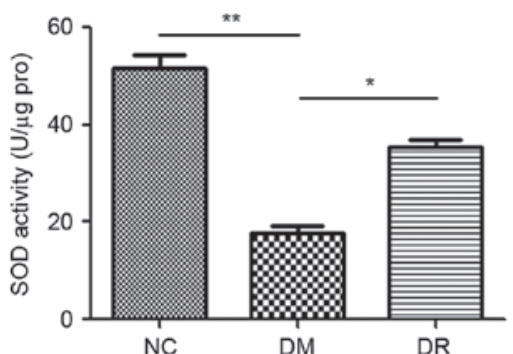

B
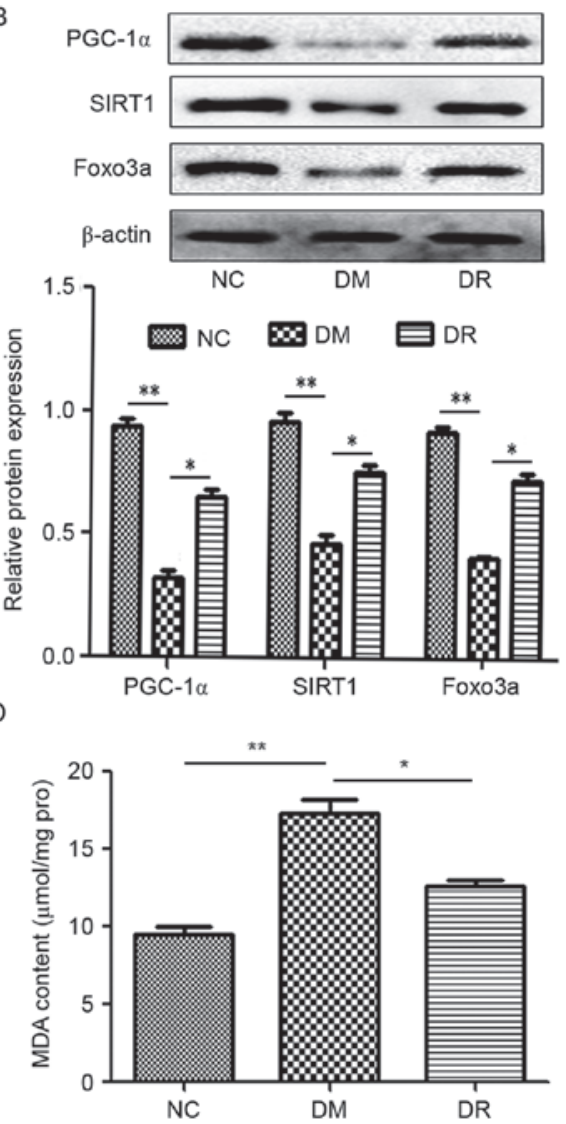

Figure 1. (A) RSV modulates PGC-1 $\alpha$, SIRT1 and FOXO3a (A) mRNA and (B) protein levels as assessed by reverse transcription-quantitative polymerase chain reaction and western blotting, respectively. $\beta$-actin was used as an internal control. Pretreatment with RSV affected the activity of (C) SOD and (D) MDA as analyzed using ELISA. "P $<0.05$ and ${ }^{* *} \mathrm{P}<0.01$. RSV, resveratrol; PGC1 $\alpha$, peroxisome proliferator-activated receptor- $\gamma$ coactivator-1 $\alpha$; SIRT1, Sirtuin 1; FOXO3a, forkhead box O3a; SOD, superoxide dismutase; MDA, malondialdehyde; NC, normal control group; DM, streptozotocin-induced diabetic group; DR, RSV treated streptozotocin-induced diabetic group; RSV, resveratrol.
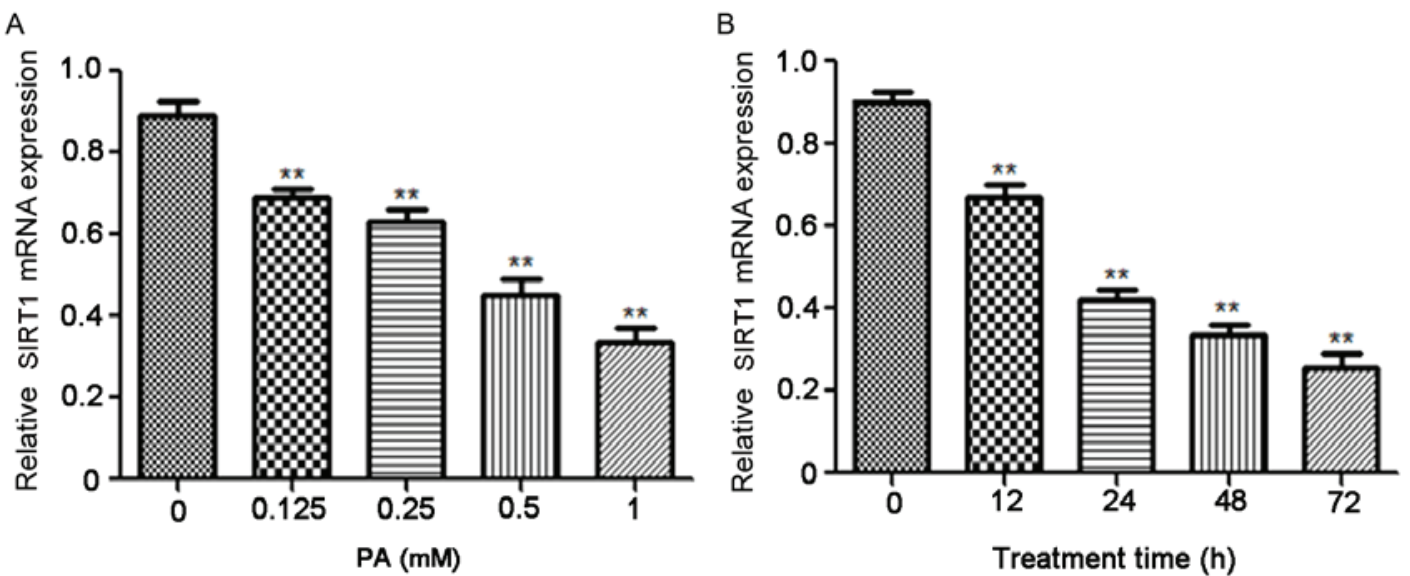

Figure 2. PA suppressed the expression of SIRT1 in a dose- and time-dependent manner. (A) INS-1E cells were treated with $0,0.125,0.25,0.5$ and $1 \mathrm{mM}$ PA for $24 \mathrm{~h}$ and the expression of SIRT1 mRNA was assessed using RT-qPCR. ${ }^{* *} \mathrm{P}<0.01$ vs. $0 \mathrm{mM} \mathrm{PA}$ (B) INS-1E cells were pretreated with $0.5 \mathrm{mM}$ PA for 0,12 , 24, 48 and $72 \mathrm{~h}$, respectively and SIRT1 mRNA was measured using RT-qPCR. $\beta$-actin was used as an internal control. ${ }^{* *} \mathrm{P}<0.01$ vs. 0 h. PA, palmitic acid; SIRT1, Sirtuin 1; INS-1E, insulinoma cell line clone 1E; RT-qPCR, reverse transcription-quantitative polymerase chain reaction.

PDX-1, MAFA and INS expression as well as the PA-induced decrease in ACC expression compared with the $\mathrm{HF}+\mathrm{RSV}+\mathrm{NC}$ siRNA group ( $\mathrm{P}<0.01 ;$ Fig. 4). These data suggest that RSV modulates the expression of NRF, mtTFA, CPT-1, LCAD, PDX-1, MAFA and INS via SIRT1 in PA-induced INS-1E cells.
RSV affects the expression of SOD and MDA via SIRT1 in PA-induced INS-1E cells. The expression of SOD and MDA mRNA in INS-1E cells following SIRT1 knockdown was assessed to determine the effect of SIRT1 on oxidative stress. Compared with the NC group, PA significantly suppressed SOD mRNA expression $(\mathrm{P}<0.01$; Fig. 5A) and RSV treatment 

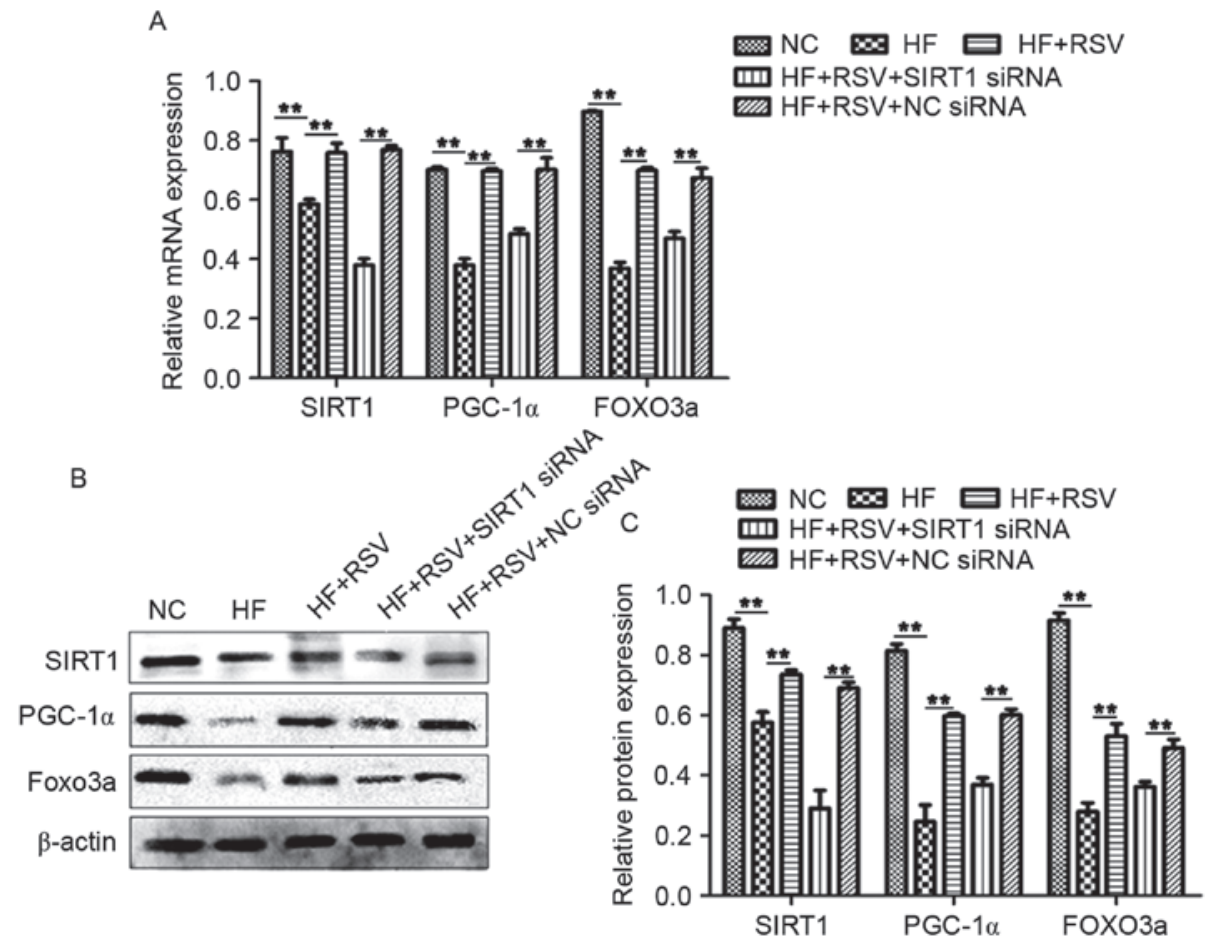

Figure 3. RSV modulates the expression of mitochondrial biogenesis-related, lipid metabolism-related and $\beta$-cells-related genes via SIRT1 in INS-1E cells. (A) mRNA and (B) protein levels of PGC-1 $\alpha$ and FOXO3a in INS-1E cells. (C) The relative densities of target protein bands were normalized against $\beta$-actin. ${ }^{* *} \mathrm{P}<0.01$. SIRT1, Sirtuin 1; PGC1 $\alpha$, peroxisome proliferator-activated receptor- $\gamma$ coactivator-1 $\alpha$; FOXO3a, forkhead box O3a; NC, normal INS-1E cells pretreated with vehicle; HF, INS-1E cells incubated with $0.5 \mathrm{mM}$ PA for $24 \mathrm{~h}$; HF+RSV, INS-1E cells incubated with $0.5 \mathrm{mM}$ PA and $10 \mu \mathrm{M}$ RSV for $24 \mathrm{~h}$; $\mathrm{HF}+\mathrm{RSV}+\mathrm{SIRT}$ siRNA, INS-1E cells transfected with SIRT1 siRNA for $48 \mathrm{~h}$ and incubated with PA $0.5 \mu \mathrm{M}$ and $10 \mu \mathrm{M}$ RSV for $24 \mathrm{~h}$; HF+RSV+NC siRNA, INS-1E cells transfected with negative control siRNA for $48 \mathrm{~h}$ and incubated with PA $0.5 \mathrm{mM}$ and $10 \mu \mathrm{M}$ RSV for $24 \mathrm{~h}$

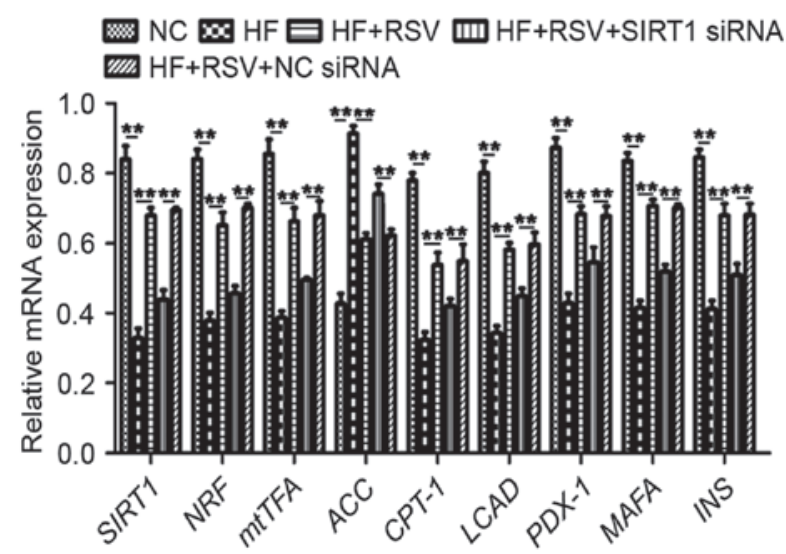

Figure 4. RSV regulated the levels of mitochondrial biogenesis-associated, lipid metabolism-associated and $\beta$-cells-associated genes via SIRT1 ${ }^{* *} \mathrm{P}<0.01$. SIRT1, Sirtuin 1; INS-1E, insulinoma cell line clone 1E; PGC1 $\alpha$ peroxisome proliferator-activated receptor- $\gamma$ coactivator- $1 \alpha$; FOXO3a, forkhead box O3a; NC, normal INS-1E cells pretreated with vehicle; HF, INS-1E cells incubated with $30 \mathrm{mM}$ PA for $24 \mathrm{~h}$; HF+RSV, INS-1E cells incubated with $30 \mathrm{mM}$ PA and $10 \mu \mathrm{m}$ RSV for $24 \mathrm{~h}$; HF+RSV+SIRT siRNA, INS-1E cells transfected with SIRT1 siRNA for $48 \mathrm{~h}$ and incubated with PA $30 \mathrm{mM}$ and $10 \mu \mathrm{m}$ RSV for $24 \mathrm{~h}$; HF+RSV+NC siRNA, INS-1E cells transfected with negative control siRNA for $48 \mathrm{~h}$ and incubated with PA $30 \mathrm{mM}$ and $10 \mu \mathrm{m} \mathrm{RSV}$ for $24 \mathrm{~h}$; NRF, nuclear respiratory factor; mtTFA, mitochondrial transcription factor A; ACC, acetyl-CoA carboxylase; CPT-1, lipid metabolism-related carnitine palmitoyltransferase 1; LCAD, long-chain acyl-CoA dehydrogenase; PDX-1, pancreatic duodenal homeobox-1; MAFA, mid-arm fat area; INS, insulin; siRNA, small interfering RNA.

significantly reversed this effect $(\mathrm{P}<0.01$; Fig. 5A). However, SIRT1 knockdown significantly decreased SOD expression compared with the HF+RSV+NC siRNA group $(\mathrm{P}<0.01$; Fig. 5A). The administration of PA significantly increased MDA expression compared with the NC group $(\mathrm{P}<0.01$; Fig. 5B) and RSV significantly attenuated this upregulation (P<0.01; Fig. 5B). SIRT1 knockdown significantly upregulated MDA mRNA expression compared with the HF+RSV+NC siRNA group $(\mathrm{P}<0.01$; Fig. $5 \mathrm{~B})$. These data indicate that RSV regulates SOD and MDA levels via SIRT1 in PA-treated INS-1E cells.

\section{Discussion}

Over the past few decades, the incidence and prevalence of DM have increased worldwide, primarily due to the increased incidence of T2DM (17). As such, there is a need to develop additional treatments and novel prevention strategies for T2DM. In the present study, a rat model of STZ-induced DM was constructed and the therapeutic effects of RSV were assessed. Furthermore, PA-induced INS-1E cells were assessed in vitro to investigate the underlying molecular mechanisms of T2DM. The results of the present study indicate that RSV mitigates the development of T2DM via SIRT1 by modulating mitochondrial biogenesis, lipid metabolism and $\beta$-cells development.

Abdominal injection of STZ is a widely used technique for DM modeling (23). In the present study, glucose and INS levels were assessed following STZ administration. It was demonstrated that glucose and INS levels were significantly upregulated, indicating that the DM model was constructed successfully. The administration of RSV attenuated the 
A

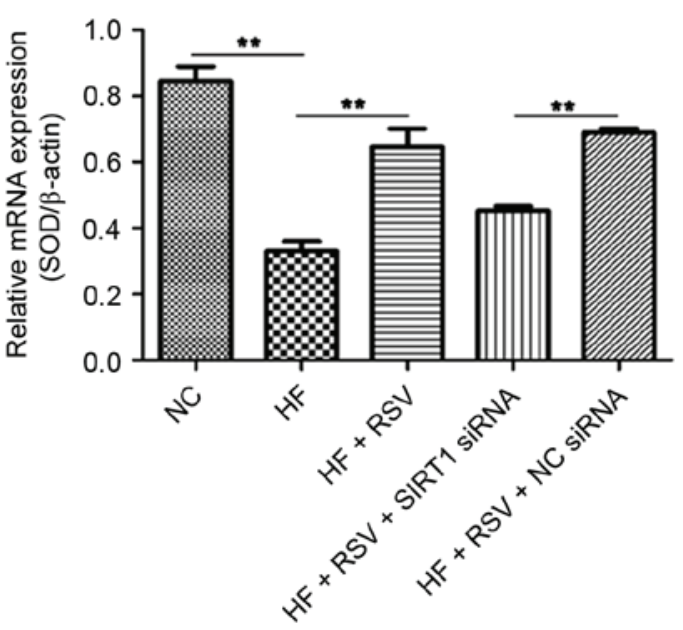

B

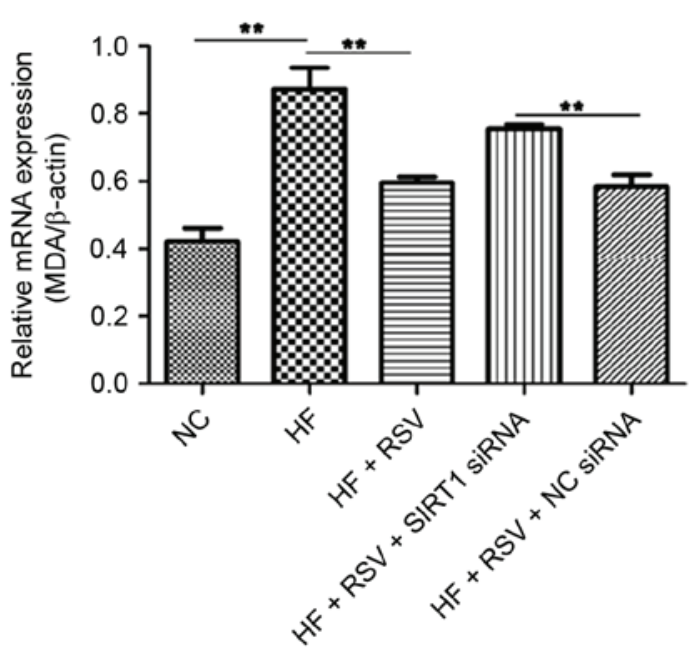

Figure 5. RSV regulates SOD and MDA mRNA levels via SIRT1 in INS-1E cells. The relative expression of (A) SOD and (B) MDA was normalized to $\beta$-actin in PA-induced INS-1E cells. * ${ }^{* *}<0.01$. RSV, resveratrol; SOD, superoxide dismutase; MDA, malondialdehyde; SIRT1, Sirtuin 1; INS-1E, insulinoma cell line clone 1E; NC, normal INS-1E cells pretreated with vehicle; HF, INS-1E cells incubated with $30 \mathrm{mM}$ PA for $24 \mathrm{~h}$; HF+RSV, INS-1E cells incubated with $30 \mathrm{mM}$ PA and $10 \mu \mathrm{m}$ RSV for $24 \mathrm{~h}$; HF+RSV+SIRT siRNA, INS-1E cells transfected with SIRT1 siRNA for $48 \mathrm{~h}$ and incubated with PA $30 \mathrm{mM}$ and $10 \mu \mathrm{m}$ RSV for $24 \mathrm{~h}$; HF+RSV+NC siRNA, INS-1E cells transfected with negative control siRNA for $48 \mathrm{~h}$ and incubated with PA $30 \mathrm{mM}$ and $10 \mu \mathrm{m}$ RSV for $24 \mathrm{~h}$; siRNA, small interfering RNA.

DM-induced alterations in glucose and INS levels, suggesting that RSV mitigates T2DM. These data were similar to those obtained from a previous study (11) and support the use of RSV as a potential glucose-lowering agent in T2DM. Furthermore, the results of an in vivo study indicated that RSV increases SIRT1 expression and stimulates PGC-1a activity in skeletal muscle (24). The results of the present study demonstrate that RSV is able to mitigate the STZ-induced inhibition of SIRT1, PGC-1 $\alpha$ and FOXO3a, which is in accordance with previous results (25-27). A number of previous studies have reported that INS resistance is associated with mitochondrial dysfunction (22) and these results may be caused by oxidative stress (28). PGC-1 $\alpha$ stimulates mitochondrial biogenesis and electron transport activity to suppress ROS production and ROS levels are also affected by MDA. Furthermore, FOXO3a serves a central role in controlling oxidative stress (29). SOD is an antioxidant enzyme and to a certain extent, SOD levels represent the level of oxidative stress (30). The results of the present study indicated that MDA activity in rats with DM was higher compared with the NC group; however, treatment with RSV reversed this effect. The opposite trend was observed in SOD activity. These data indicate that there is an association between DM and oxidative stress and that RSV is able to successfully attenuate these conditions.

INS secretion from pancreatic islet $\beta$-cells is regulated by a number of factors; an increase in blood glucose is the predominant trigger; however, fatty and amino acids may also act as direct or indirect stimuli (31). It has been reported that PA induces lipotoxicity in rat INS-producing cells (32). In the present study, PA was used to induce INS secretion in INS-1E cells. The results demonstrated that PA suppressed the expression of SIRT1 in a dose- and time-dependent manner, suggesting that SIRT1 serves an important role in the regulation of lipid metabolism. Furthermore, PA inhibited PGC-1 $\alpha$ and FOXO3a expression. SIRT1 knockdown attenuated the effects of RSV on PA-inhibited PGC-1a and FOXO3a expression. It has previously been reported that SIRT1 mediates the deacetylation of certain targets, including PGC-1 $\alpha$, FOXO3a and p53, in mitochondrial biogenesis, lipid metabolism and inflammation (33). The results of the present study suggest that RSV functions via SIRT1 in PA-induced INS-1E cells. SIRT1 regulates a range of cellular functions affecting metabolic homeostasis and the link between metabolism and INS secretion depends on mitochondrial function (34). PGC-1 $\alpha$ is a mitochondrial regulator that serves an essential role in cellular energy metabolism and the ectopic expression of PGC-1 $\alpha$ induces the expression of NRFs and mtTFA prior to mitochondrial biogenesis (35). The results of the present study demonstrate that SIRT1 knockdown modulates NRFs and mtTFA levels in cells treated with PA and RSV. These data suggest that RSV functions via SIRT1 to modulate mitochondrial biogenesis.

It has been suggested that INS resistance occurs following disturbances in lipid metabolism (36). Therefore, the current study investigated whether RSV affects lipid metabolism. It was demonstrated that SIRT1 knockdown altered the effect of RSV on ACC, CPT-1 and LCDA expression. A previous study reported that SIRT1 suppresses the expression of ACC to reduce lipogenesis and upregulates CPT1 and LCAD to increase normal $\beta$-oxidation activities (37). Furthermore, it was demonstrated that RSV modulates ACC, CPT-1 and LCDA activity in lipid metabolism via SIRT1, suggesting a novel link between RSV, T2DM and lipid metabolism. PDX-1, MAFA and INS contribute to pancreas development, $\beta$-cells differentiation and the maintenance of mature $\beta$-cells function (38). In the present study, SIRT1 knockdown regulated levels of PDX-1, MAFA and INS mRNA. These results suggest that RSV may affect $\beta$-cells functionality via SIRT1.

In conclusion, the results of the present study suggest that RSV modulates the expression of SIRT1, PGC-1 $\alpha$ and FOXO3a in a rat model of STZ-injected DM. In vitro, $\mathrm{RSV}$ significantly affected levels of PGC- $1 \alpha$ and FOXO3a via 
SIRT1. RSV promoted mitochondrial biogenesis to stimulate INS secretion and enhanced fatty acid $\beta$-oxidation activity to reduce ectopic lipid deposition and promote INS expression. The present study provides an insight into the mechanism by which RSV prevents T2DM via SIRT1 and provides evidence that RSV may be used as a clinical treatment to prevent the development of T2DM.

\section{Acknowledgements}

The present study was supported by the Research Fund for Clinical Medicine, Chinese Medical Association (grant no. 13050770462), the Heilongjiang Postdoctoral Science Foundation (grant no. LBH-Z15159), the Health Department of Heilongjiang Province (grant no. 2016-022), the Scientific Foundation of the First Affiliated Hospital of Harbin Medical University (grant nos. 2016B008 and 2017B001), the Technology Innovation Program of Harbin City (grant no. 2016RAQXJ167), the National Natural Science Foundation of China (grant no. 81370929)) and the Innovative Science Research Project of Harbin Medical University (grant nos. 2016LCZX40 and 2016LCZX51).

\section{References}

1. Franz MJ, Boucher JL, Rutten-Ramos S and VanWormer JJ: Lifestyle weight-loss intervention outcomes in overweight and obese adults with type 2 diabetes: A systematic review and meta-analysis of randomized clinical trials. J Acad Nutr Diet 115: $1447-1463,2015$

2. Galling B, Roldan A, Nielsen RE, Nielsen J, Gerhard T, Carbon M, Stubbs B, Vancampfort D, De Hert M, Olfson M, et al: Type 2 diabetes mellitus in youth exposed to antipsychotics: A systematic review and meta-analysis. JAMA Psychiatry 73: 247-259, 2016.

3. Dart AB, Martens PJ, Rigatto C, Brownell MD, Dean HJ and Sellers EA: Earlier onset of complications in youth with type 2 diabetes. Diabetes Care 37: 436-443, 2014.

4. Liberopoulos EN, Tsouli S, Mikhailidis DP and Elisaf MS: Preventing type 2 diabetes in high risk patients: An overview of lifestyle and pharmacological measures. Curr Drug Targets 7: 211-228, 2006.

5. Lotfy M, Adeghate J, Kalasz H, Singh J and Adeghate E: Chronic complications of diabetes mellitus: A mini review. Curr Diabetes Rev 13: 3-10, 2017.

6. Saisho Y: $\beta$-cell dysfunction: Its critical role in prevention and management of type 2 diabetes. World J Diabetes 6: 109-124, 2015.

7. Lomonaco R, Bril F, Portillo-Sanchez P, Ortiz-Lopez C, Orsak B, Biernacki D, Lo M, Suman A, Weber MH and Cusi K: Metabolic impact of nonalcoholic steatohepatitis in obese patients with type 2 diabetes. Diabetes Care 39: 632-638, 2016.

8. Lin YL, Lai YH, Wang CH, Kuo CH, Liou HH and Hsu BG: Triceps skinfold thickness is associated with lumbar bone mineral density in peritoneal dialysis patients. Ther Apher Dial 21: 102-107, 2017.

9. Morino K, Petersen KF, Dufour S, Befroy D, Frattini J, Shatzkes N, Neschen S, White MF, Bilz S, Sono S, et al: Reduced mitochondrial density and increased IRS-1 serine phosphorylation in muscle of insulin-resistant offspring of type 2 diabetic parents. J Clin Invest 115: 3587-3593, 2005.

10. Hausenblas HA, Schoulda JA and Smoliga JM: Resveratrol treatment as an adjunct to pharmacological management in type 2 diabetes mellitus-systematic review and meta-analysis. Mol Nutr Food Res 59: 147-159, 2015.

11. Wicklow B, Wittmeier K, T' Jong GW, McGavock J, Robert M, Duhamel T and Dolinsky VW: Proposed trial: Safety and efficacy of resveratrol for the treatment of non-alcoholic fatty liver disease (NAFLD) and associated insulin resistance in adolescents who are overweight or obese adolescents-rationale and protocol. Biochem Cell Biol 93: 522-530, 2015.
12. Szkudelski T and Szkudelska K: Resveratrol and diabetes: From animal to human studies. Biochim Biophys Acta 1852: 1145-1154, 2015.

13. Guarente L and Picard F: Calorie restriction-the SIR 2 connection. Cell 120: 473-482, 2005.

14. McBurney MW, Clark-Knowles KV, Caron AZ and Gray DA: SIRT1 is a highly networked protein that mediates the adaptation to chronic physiological Stress. Genes Cancer 4: 125-134, 2013.

15. Song R, Xu W, Chen Y, Li Z, Zeng Y and Fu Y: The expression of Sirtuins 1 and 4 in peripheral blood leukocytes from patients with type 2 diabetes. Eur J Histochem 55: e10, 2011.

16. Han J, Wei M, Wang Q, Li X, Zhu C, Mao Y, Wei L, Sun Y and Jia W: Association of genetic variants of SIRT1 with type 2 diabetes mellitus. Gene Expr 16: 177-185, 2015.

17. Kitada M and Koya D: SIRT1 in type 2 diabetes: Mechanisms and therapeutic potential. Diabetes Metab J 37: 315-325, 2013.

18. Lovis P, Gattesco S and Regazzi R: Regulation of the expression of components of the exocytotic machinery of insulin-secreting cells by microRNAs. Biol Chem 389: 305-312, 2008.

19. Nathan DM, Buse JB, Davidson MB, Heine RJ, Holman RR, Sherwin R, Zinman B; Professional Practice Committee, American Diabetes Association; European Association for the Study of Diabetes: Management of hyperglycaemia in type 2 diabetes: A consensus algorithm for the initiation and adjustment of therapy. A consensus statement from the American Diabetes Association and the European Association for the Study of Diabetes. Diabetologia 49: 1711-1721, 2006.

20. Banin RM, Hirata BK, Andrade IS, Zemdegs JC, Clemente AP, Dornellas AP, Boldarine VT, Estadella D, Albuquerque KT, Oyama LM, et al: Beneficial effects of Ginkgo biloba extract on insulin signaling cascade, dyslipidemia, and body adiposity of diet-induced obese rats. Braz J Med Biol Res 47: 780-788, 2014.

21. Livak KJ and Schmittgen TD: Analysis of relative gene expression data using real-time quantitative PCR and the 2(-Delta Delta C(T)) method. Methods 25: 402-408, 2001.

22. Tangvarasittichai S: Oxidative stress, insulin resistance, dyslipidemia and type 2 diabetes mellitus. World J Diabetes 6: 456-480, 2015.

23. Marangoni MN, Brady ST, Chowdhury SA and Piano MR: The co-occurrence of myocardial dysfunction and peripheral insensate neuropathy in a streptozotocin-induced rat model of diabetes. Cardiovasc Diabetol 13: 11, 2014.

24. Goh KP, Lee HY, Lau DP, Supaat W, Chan YH and Koh AF: Effects of resveratrol in patients with type 2 diabetes mellitus on skeletal muscle SIRT1 expression and energy expenditure. Int J Sport Nutr Exerc Metab 24: 2-13, 2014.

25. Huang XZ, Wen D, Zhang M, Xie Q, Ma L, Guan Y, Ren Y, Chen J and Hao CM: Sirtl activation ameliorates renal fibrosis by inhibiting the TGF- $\beta /$ Smad3 pathway. J Cell Biochem 115: 996-1005, 2014.

26. Higashida K, Kim SH, Jung SR, Asaka M, Holloszy JO and Han DH: Effects of resveratrol and SIRT1 on PGC-1 $\alpha$ activity and mitochondrial biogenesis: A reevaluation. PLoS Biol 11: e1001603, 2013.

27. Song X, Yang B, Qiu F, Jia M and Fu G: High glucose and free fatty acids induce endothelial progenitor cell senescence via PGC-1 $\alpha /$ SIRT1 signaling pathway. Cell Biol Int 41: 1146-1159, 2017.

28. Zhang HH, Ma XJ, Wu LN, Zhao YY, Zhang PY, Zhang YH, Shao MW, Liu F, Li F and Qin GJ: SIRT1 attenuates high glucose-induced insulin resistance via reducing mitochondrial dysfunction in skeletal muscle cells. Exp Biol Med (Maywood) 240: 557-565, 2015.

29. Brunet A, Sweeney LB, Sturgill JF, Chua KF, Greer PL, Lin Y, Tran H, Ross SE, Mostoslavsky R, Cohen HY, et al: Stress-dependent regulation of FOXO transcription factors by the SIRT1 deacetylase. Science 303: 2011-2015, 2004.

30. Liu H, Sheng M, Liu Y, Wang P, Chen Y, Chen L, Wang W and $\mathrm{Li}$ B: Expression of SIRT1 and oxidative stress in diabetic dry eye. Int J Clin Exp Pathol 8: 7644-7653, 2015.

31. Tan C, Voss U, Svensson S, Erlinge D and Olde B: High glucose and free fatty acids induce beta cell apoptosis via autocrine effects of ADP acting on the $\mathrm{P} 2 \mathrm{Y}(13)$ receptor. Purinergic Signal 9: 67-79, 2013.

32. Plotz T, Hartmann M, Lenzen S and Elsner M: The role of lipid droplet formation in the protection of unsaturated fatty acids against palmitic acid induced lipotoxicity to rat insulin-producing cells. Nutr Metab (Lond) 13: 16, 2016. 
33. Ghinis-Hozumi Y, González-Dávalos L, Antaramian A, Villarroya F, Piña E, Shimada A, Varela-Echavarría A and Mora O: Effect of resveratrol and lipoic acid on sirtuin-regulated expression of metabolic genes in bovine liver and muscle slice cultures. J Anim Sci 93: 3820-3831, 2015.

34. Wiederkehr A and Wollheim CB: Mitochondrial signals drive insulin secretion in the pancreatic $\beta$-cell. Mol Cell Endocrinol 353: 128-137, 2012.

35. Chen $\mathrm{CH}$, Nagayama K, Enomoto N, Miyasaka Y, Kurosaki M, Sakamoto N, Maekawa S, Kakinuma S, Ikeda T, Izumi N, et al: Enhancement of mitochondrial gene expression in the liver of primary biliary cirrhosis. Hepatol Res 31: 24-30, 2005.

36. Chen S, Zhao X, Ran L, Wan J, Wang X, Qin Y, Shu F, Gao Y, Yuan L, Zhang Q and Mi M: Resveratrol improves insulin resistance, glucose and lipid metabolism in patients with non-alcoholic fatty liver disease: A randomized controlled trial. Dig Liver Dis 47: 226-232, 2015.
37. Chen LL, Zhang HH, Zheng J, Hu X, Kong W, Hu D, Wang SX and Zhang P: Resveratrol attenuates high-fat diet-induced insulin resistance by influencing skeletal muscle lipid transport and subsarcolemmal mitochondrial $\beta$-oxidation. Metabolism 60: 1598-1609, 2011

38. Kaneto H, Miyatsuka T, Kawamori D, Yamamoto K, Kato K, Shiraiwa T, Katakami N, Yamasaki Y, Matsuhisa M and Matsuoka TA: PDX-1 and MafA play a crucial role in pancreatic beta-cell differentiation and maintenance of mature beta-cell function. Endocr J 55: 235-252, 2008. 